\title{
Utilização de Six Sigma na Melhoria de Processos de Software Alinhados ao Planejamento Estratégico - Um Caso Prático da Dell
}

\author{
Andressa Covatti, Jorge Luis Nicolas Audy. \\ Faculdade de Informática - Pontifícia Universidade Católica do Rio Grande do Sul \\ Porto Alegre - RS - Brazil \\ \{acovatti,audy\}@inf.pucrs.br
}

\begin{abstract}
Software quality improvements for software development process are very important for organizations that are implementing quality programs. The various models and methodologies offers by the market need to be used to contribute for the process improvements implementation to avoid rework and cost increase. Besides that, the idea is to have these process improvements programs aligned with the organizational strategic planning. The integration among BSC, CMMI and Six Sigma can help the organization to work in software process improvement using Six Sigma to improve process created based on CMMI and aligned with the organizational strategic planning according the practical case that is being showed in this work.
\end{abstract}

Resumo. A melhoria dos processos de desenvolvimento de software são fundamentais para as organizações que implementam programas de qualidade. Os diversos modelos e metodologias oferecidos pelo mercado precisam ser utilizados de forma a contribuir para a implementação dessas melhorias sem causar retrabalho e aumento de custos para a organização. Além disso, o ideal é que os programas de melhoria estejam alinhados com os objetivos estratégicos da organização. Dessa forma a utilização do BSC, CMMI e Six Sigma de forma integrada pode ajudar a organização a trabalhar em melhorias de processo de software utilizando o Six Sigma para melhorar os processos criados com base no CMMI e de forma alinhada ao planejamento estratégico da organização conforme mostra o caso prático descrito nesse artigo.

\section{Introdução}

A melhoria continua dos processos de desenvolvimento de software é um objetivo das organizações que adotam os programas de qualidade. A preocupação com o processo de software está fortemente relacionada à necessidade de entender, avaliar, aprender, melhorar, planejar, verificar e principalmente controlar o trabalho dos engenheiros de software. Para atingir essa meta é preciso: documentar, definir, medir, analisar, avaliar, controlar e alterar os processos de desenvolvimento. Esses controles poderão ajudar a organização a reduzir prazos, fornecer orçamentos confiáveis, acelerar seus processos de desenvolvimento e terão com certeza a primazia do mercado [Rocha, Maldonado e Weber 2001].

O processo de melhoria de processos deve ser iterativo e de longo prazo, ou seja, além de implementar processos de desenvolvimento nas organizações é preciso medi-los, estipular limites e melhorá-los no decorrer do tempo, nesse contexto as iniciativas BSC, CMMI e Six 
Sigma, podem ser chamadas de complementares, no momento que utilizamos o CMMI para implementação e institucionalização de processos de desenvolvimento e o Six Sigma como a metodologia para implementação do processo de melhoria contínua. Juntando a isso a utilização do BSC para ajudar o programa de melhoria a estar alinhado com os objetivos estratégicos da organização e o GQM para definição de boas métricas.

O objetivo desse artigo é apresentar o modelo de referência e o método que propõe a integração entre essas iniciativas e, além disso, relatar um caso prático dessa aplicação na Dell Inc. o artigo está organizado da seguinte forma: na seção 2 (dois) será apresentada a base teórica utilizada. A seção 3 (três) apresenta o método de integração proposto. Na seção 4 (cinco) é apresentado um caso prático. Ao final, na seção 5 (cinco), são apresentadas as conclusões e as lições aprendidas.

\section{Melhoria de Processo de Software}

\subsection{CMMI}

O CMMI (Capability Maturity Model Integrated) é uma evolução do modelo CMM [Kulpa 2003]. Desde o início dos anos 90 diversos CMMs foram desenvolvidos para serem utilizados em várias disciplinas distintas. O CMMI foi criado para ajudar as organizações nas avaliações de maturidade ou capacidade das áreas de processo, estabelecer prioridades de melhoria e implementar essas melhorias.

Atualmente quatro áreas estão cobertas pelo CMMI: Engenharia de Sistemas, Engenharia de Software, Desenvolvimento Integrado de Produtos e Processos, Fornecedores [Chrissis, Konrad, Shrum 2003].

\subsection{Six Sigma}

A metodologia Six Sigma pode ser considerada uma filosofia para o gerenciamento da qualidade. É uma metodologia para gerenciar a melhoria de negócios e processos. Essa metodologia tem o foco no cliente e utiliza-se de dados para direcionar e implementar soluções encontradas a partir da análise de dados reais, para um determinado problema ou objetivos.

O método utiliza-se de estatística para medir e analisar os processos de uma organização. Estatisticamente falando atingir o Six Sigma significa que o processo ou produto é realizado ou fabricado com quase nenhum defeito, o Six Sigma representa 3,4 defeitos encontrados por milhão de oportunidade, o que representa uma potencialidade de aproximadamente 99,9996\% de eficiência. Sabe-se que hoje em dia a maioria dos processos implantados opera entre 3 e 4 sigma, ou seja, aproximadamente entre 93,3\% e 99,4\% de eficiência [Hayes 2005]].

O Six Sigma diferentemente dos modelos de qualidade é uma metodologia orientada ao negócio que utiliza uma abordagem de melhoria de processo com vários aspectos, redução de custos e aumento de lucros. Seu principio fundamental é o aumento da satisfação do cliente baseado na redução de defeitos. A metodologia do Six Sigma, quando utilizada pelas organizações, permite que o time encarregado das ações e implementações de melhorias identificar o processo e as medidas correspondentes que será afetado. 


\subsection{Balanced Scorecard (BSC)}

As organizações se deparam com diversos obstáculos quando desenvolvem um sistema de medição que possa medir as coisas certas nas horas certas. O que se precisa é um sistema que balanceie a histórica precisão dos números financeiros com os objetivos de desempenho futuro enquanto também ajuda a organização na implementação de diferentes estratégias.

O BSC é uma ferramenta que atende aos dois propósitos, a metodologia desenvolvida por Robert Kaplan e David Norton em resultado a uma pesquisa nos anos 90 pretendia provar que apenas indicadores financeiros eram ineficientes para as organizações globais. Através de pesquisa realizada em diversas empresas, eles criaram o que chamaram de Balanced Scorecard, que além dos indicadores financeiros também se preocupa com os problemas de clientes, de processos internos e de aprendizagem e crescimento [Niven 2002].

O BSC é um sistema de avaliação de desempenho empresarial reconhecido pela indústria para medir a saúde das organizações. Ele pode ser utilizado como uma ferramenta de desenvolvimento que traduz os objetivos e metas da organização em um conjunto compreensível de medidas que fornecem um conjunto de medidas consistentes ao sistema gerencial [Goethert e Fisher 2003].

\section{MIBCIS - Modelo de Referência e Método Proposto}

Após o estudo de vários modelos, métodos e metodologias; torna-se claro que os esforços para implementação de processos e programas de melhoria continuam na área de qualidade nas organizações de desenvolvimento de software precisam estar alinhadas ao planejamento estratégico da organização.

Podemos observar na figura 1 um modelo de referência construído com o objetivo de construir o método proposto. Este modelo de referência mostra a integração do BSC, CMMI e Six Sigma é vista nos diversos níveis das organizações e também podemos observar que o GQM poderá suportar a definição e análise de métricas em qualquer um desses níveis.

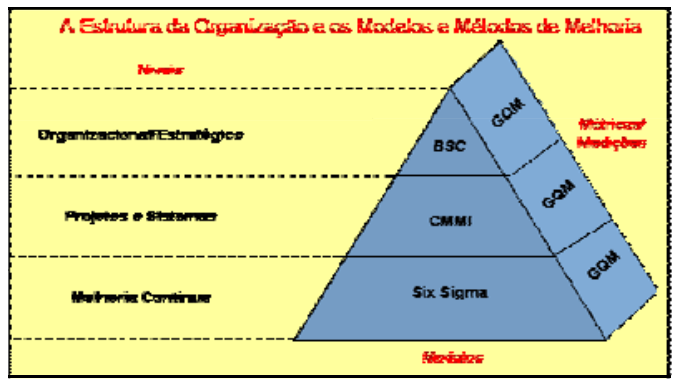

Figura 1: A integração do BSC, CMMI e Six Sigma nos diversos níveis organizacionais.

O método MIBCIS - Método de Integração entre o BSC, CMMI e Six Sigma utilizando GQM no suporte a definição de métricas, sugere às organizações que o programa de qualidade e melhoria de processos seja implantado de forma alinhada aos objetivos estratégicos da mesma. Na figura 2 podemos observar o método proposto, assim como, também podemos observar que as atividades propostas não acontecem em seqüência. Algumas atividades acontecem em períodos distintos no tempo, como o planejamento estratégico, que é feito para os próximos 5 ou 10 anos e revisado ano-a-ano. Outras 
atividades, como a definição de novas métricas, podem se repetir cada vez que a organização precisar de uma nova métrica. Assim como a definição de novos processos para o desenvolvimento de projetos pode ser necessária a qualquer momento. O ciclo de melhoria contínua não deve ter fim, os indicadores, métricas e processos das organizações precisam ser constantemente avaliados e melhorados. A proposta mais importante desse método, é que todas essas atividades (iniciativas) aconteçam na organização de forma sincronizada e com o objetivo de melhorar a qualidade do produto e aumentar a satisfação do cliente.

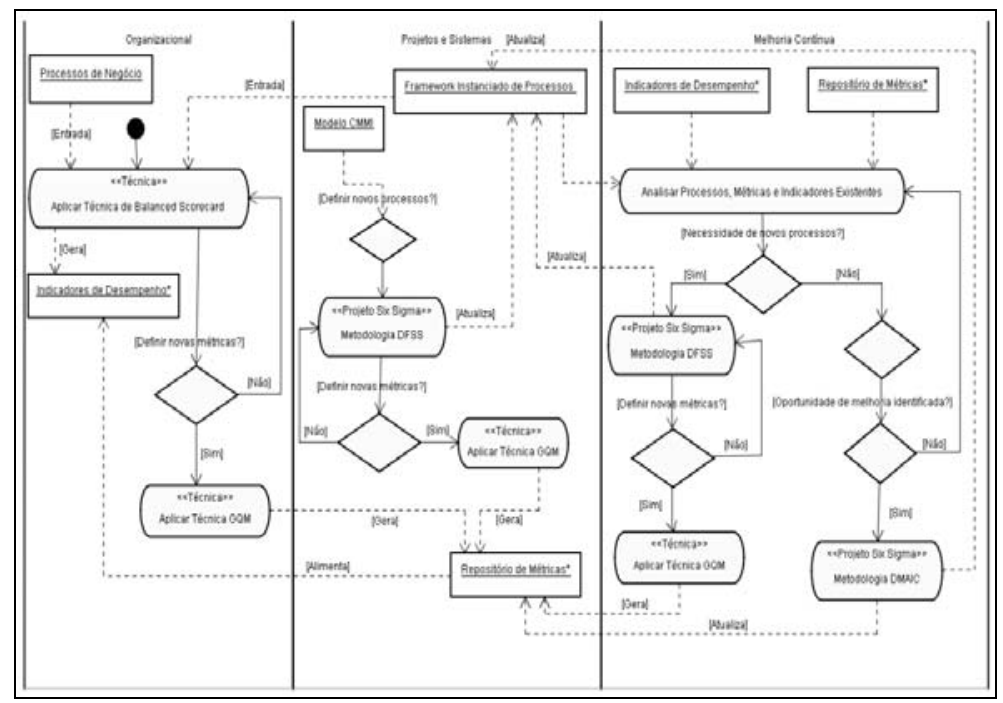

Figura 2: O método - MIBCIS - Método de Integração entre o BSC, CMMI e Six Sigma utilizando GQM no suporte a definição de métricas.

Nível Organizacional: no método proposto, assim como no modelo de referência, o BSC é utilizado no nível estratégico das organizações onde a partir dos processos de negócio os grandes objetivos estratégicos a alcançar são definidos, além disso, o método propõe a utilização do GQM sempre que novas métricas sejam necessárias nesse nível.

Nível de Projetos e Sistemas: o método propõe a utilização do CMMI para definir os processos que serão utilizados pela organização faz com que a mesma trabalhe com base nas melhores práticas encontradas no modelo para desenvolver a sua própria base de processos, o que ajudará a identificar benefícios mensuráveis a partir da melhoria contínua de processos.

Nível de Melhoria Contínua: a metodologia Six Sigma por sua vez tem por objetivo ajudar na solução de problemas complexos por meio de métodos e ferramentas quantitativas, focando em resultados financeiros e no cliente.

O método ainda propõe que a técnica GQM seja utilizada como suporte na definição de métricas e objetivos de medição que suportem o método proposto em qualquer nível de aplicação.

\section{Aplicação do MIBCIS: $O$ caso da Dell.}

A Dell Inc. possui escritórios em 34 países em todo o mundo incluindo o Brasil. De acordo com informações fornecidas pela organização, ela possui cerca de 55.000 funcionários distribuídos nesses escritórios. O projeto apresentado nesse relato de experiência apresenta os 
resultados de um projeto Six Sigma que foi desenvolvido envolvendo três regiões: Estados Unidos, Brasil e Índia.

A organização caracteriza-se por trabalhar em um ambiente de desenvolvimento de software geograficamente distribuído e a mesma está implementando e utilizando processos de desenvolvimento de software em nível global baseados nas boas práticas do SW-CMM e CMMI. A seguir algumas definições importantes para ajudar no entendimento do projeto em questão:

Framework de Processos de Desenvolvimento: a organização está trabalhando na globalização de seus processos de desenvolvimento de software, ou seja, todas as unidades que trabalham com desenvolvimento de software utilizam o mesmo processo de desenvolvimento, os mesmos artefatos (modelos) e a mesma linguagem independente da localização geográfica que se encontram. Para ajudar na globalização dos processos de desenvolvimento, um framework de processos foi desenvolvido, este contou com a contribuição e trabalho de mais de 400 funcionários da área de tecnologia em todo o mundo para escrever e revisar os processos.

Esse framework segue as boas práticas do SW-CMM e do CMMI, apesar de a organização não estar buscando nenhum nível de maturidade nos modelos ou certificação oficial. Ao framework foi dado o nome de Horizon e este já foi implantado em todas as unidades de TI da organização.

Melhoria de Processo: a organização em questão tem um programa chamado BPI (Business Process Improvement), o qual trata das melhorias de processo em toda a organização e não somente em TI. Esses projetos chamados BPI são baseados na metodologia Six Sigma, a organização somente adicionou uma fase para relatar os resultados no final dos ciclos de vida, então o ciclo de melhoria de processo do Six Sigma DMAIC nesta organização chama-se DMAICR.

Esse programa tem uma grande visibilidade e suporte por parte da gerência sênior. Cada unidade tem um objetivo financeiro para o ano que precisa ser atingido, assim como os melhores projetos também são reconhecidos em um dia chamado WQD (World Quality Day), onde todos os funcionários que tem seus projetos selecionados viajam para os Estados Unidos para apresentá-los ao maior acionista da empresa e seus executivos.

Planejamento Stratégico: O Hoshin kanri ou Hoshin plan como é normalmente chamado é uma abordagem utilizada por algumas organizações para fazer os seus planejamentos estratégicos. Uma de suas características é o planejamento, implementação e revisão feitos passo a passo quando mudanças nos processos de negócio são propostas. Essa técnica é considerada equivalente ao Balanced Scorecard, pois seu objetivo também é trabalhar os objetivos estratégicos da organização.

Um dos objetivos da organização para os anos de 2005/2006 era melhorar a produtividade no desenvolvimento de software em seus centros de desenvolvimento no Brasil e na Índia. Para maximizar as chances de sucesso nessa iniciativa de melhorar a produtividade, foi decidido fazer um projeto Six Sigma com o objetivo de descobrir quais fatores estavam impactando a produtividade e depois melhora-la. A melhoria na métrica de produtividade impactaria em 3 fatores importantes para o planejamento estratégico: (i) 
melhoria da experiência com o cliente (interno e externo), (ii) alinhamento com os demais objetivos da organização e (iii) melhoria no uso dos recursos de TI.

\subsection{O projeto DMAIC}

Fase Definir: nessa fase de definição se deu a estruturação do projeto. Um executivo nos Estados Unidos assumiu a função de executive belt para esse projeto com o objetivo de suportar as necessidades dos times ao redor do mundo. $\mathrm{O}$ time em si foi formado por 1 Green Belt e 2 Yellow Belts no Brasil e 1 Green Belt e 4 Yellow Belts na Índia. O projeto estava distribuído globalmente e tinha o foco na área de desenvolvimento no Brasil e na Índia e seu objetivo inicial era reduzir o re-trabalho e o tempo de espera entre um projeto e outro em $25 \%$ (esses dois fatores foram apontados como possíveis causas para a baixa produtividade).

Fase Medir: nessa fase os times do projeto selecionaram alguns fatores que influenciam o cálculo da produtividade, os fatores selecionados foram: re-trabalho, tempo de espera entre projetos, disponibilidade de recursos, qualidade de requisitos, tipo de projetos e habilidades necessárias para um projeto. Apesar dos vários fatores investigados, re-trabalho e tempo de espera entre projetos foram selecionados para serem trabalhados nesse projeto. A principal razão para essa escolha, além de serem causas para o problema, é que a organização não possuía dados para avaliar e fazer as possíveis correlações com os demais fatores listados.

O próximo passo foi estabelecer como os fatores re-trabalho e tempo de espera entre projetos seriam medidos, fazer com que toda a organização coletasse essas métricas da mesma forma e estabelecer a linha de base inicial, ou seja, medir o desempenho atual da produtividade na organização. Os dados de linha de base de re-trabalho e tempo de espera entre projetos podem ser observados nas figuras 3 e 4 . Na medida do tempo de espera entre projetos os valores encontrados para a média e o desvio padrão foram: 9,20 e 2,29 horas respectivamente. Já para a medida de re-trabalho, a média e o desvio padrão encontrados foram de: 15,23 14,44 horas respectivamente.

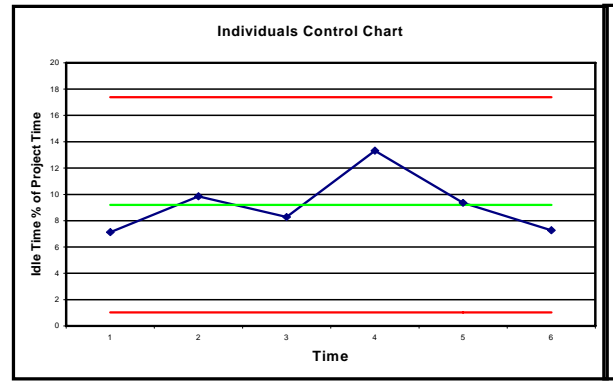

Figura 3: Tempo de Espera entre projetos - Gráfico de Controle

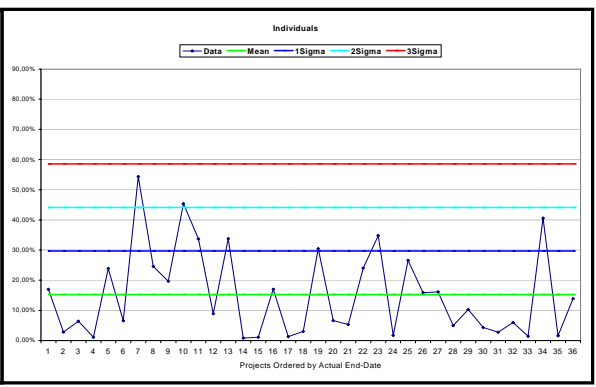

Figura 4: Re-trabalho - Gráfico de Controle.

Fase Analisar: durante essa fase os dados coletados foram analisados e correlacionados de acordo com os seguintes critérios: localização do centro, área de negócio e programas (principais 5 programas). Chegou-se a necessidade dessas correlações a partir da análise dos dados coletados. Um exemplo da análise feita pode ser observado nas figuras 5 e 6 que representam a análise do problema de tempo de espera entre projetos nos centros do Brasil e da India. 


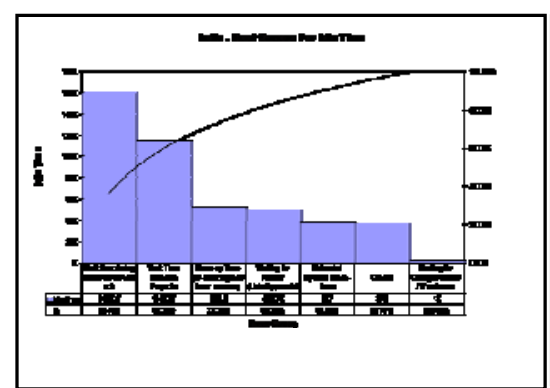

Figura 5: Análise de tempo de espera na Índia - Gráfico de Pareto.

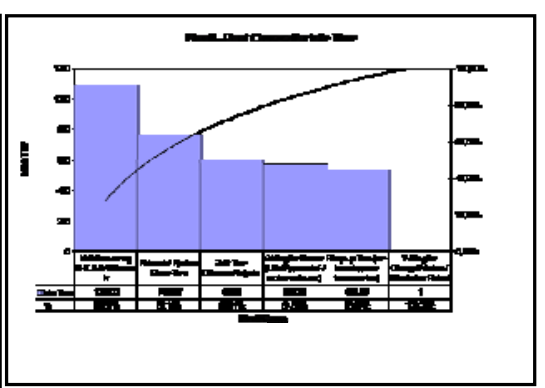

Figura 6: Análise de tempo de espera no Brasil Gráfico de Pareto.

Além dos gráficos, na fase de analisar também foram construídos diagramas de casa e efeito e conduzidos brainstormings com as pessoas envolvidas.

Fase de Melhoria (Improve): nessa fase, ações foram implementadas com o objetivo de melhorar cada uma das causas encontradas. As ações e suas respectivas causas podem ser observadas na tabela 1 :

Tabela 1. Relação entre Causas e Ações.

\begin{tabular}{|l|l|}
\hline Causas Raiz & Melhorias \\
\hline $\begin{array}{l}\text { Tempo de espera entre SIT/UAT } \\
\text { nos projetos }\end{array}$ & $\begin{array}{l}\text { Novo processo de planejamento de recursos foi criado e institucionalizado com o objetivo planejar a } \\
\text { alocação dos recursos com base nas fases dos projetos. }\end{array}$ \\
\hline $\begin{array}{l}\text { Teste unitário pouco efetivo e Teste } \\
\text { unitário e de integração com } \\
\text { estimativas pouco precisas }\end{array}$ & $\begin{array}{l}\text { Melhorias nos processos de estimativas para desenvolvimento e teste foram desenvolvidas e } \\
\text { implantadas. }\end{array}$ \\
\hline $\begin{array}{l}\text { Falta de motivação e compromisso } \\
\text { por parte dos desenvolvedores } \\
\text { especialistas, etc ...). Análise da possibilidade da utilização de Test Driven Development } \\
\text { Criação de um treinamento para desenvolvedores sobre teste unitário (todo o time foi treinado) }\end{array}$ & $\begin{array}{l}\text { Implementação do programa “Desenvolvedor Samurai” com o objetivo de: } \\
\text { Reduzir o número de defeitos no código, focando no compromisso do time de desenvolvimento; } \\
\text { Criar uma campanha de marketing com foco no "zero defeito", reforçando que código com defeito não é } \\
\text { aceitável; } \\
\text { Criação de um programa de reconhecimento. } \\
\text { Criar uma lista com os melhores desenvolvedores e compartilhar as melhores práticas utilizadas por eles. }\end{array}$ \\
\hline $\begin{array}{l}\text { Alteração de requisitos e requisitos } \\
\text { não claramente definidos }\end{array}$ & $\begin{array}{l}\text { Treinamento de requisitos, vindo de um outro projeto Six Sigma, ministrado para todos os membros } \\
\text { envolvidos no processo de desenvolvimento de requisitos nos dois centros. }\end{array}$ \\
\hline
\end{tabular}

Fase de Controle e Report: nessas fases podemos observar a melhoria ocorrida nas duas métricas de re-trabalho e tempo de espera. A redução ocorrida na métrica de re-trabalho pode ser observado nas figuras 7 e 8 , a linha vermelha mostra a média diminuiu e os processos melhoraram após a implementação das melhorias.

Além disso, se compararmos, os dados que tínhamos como linha de base com os dados coletados após a melhoria dos processos podemos observar que o desvio padrão diminuiu de 14,29 para 8,14 e que a média diminuiu de 14,44 para 8,47, o que significa uma melhoria significativa no processo, o que atendeu o objetivo de diminuir as horas de re-trabalho na organização. No que diz respeito a tempo de espera o objetivo era de melhorar em $5 \%$ e o projeto conseguiu melhorar essa métrica em 7,57\%. A média no Brasil melhorou de 6,58 para 4,93 e na Índia de 9,36 para 6,75. 


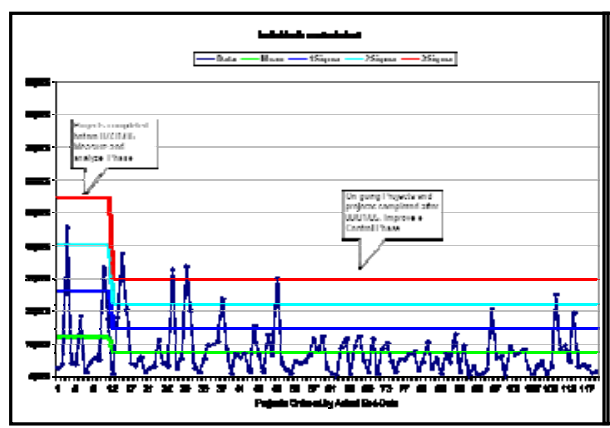

Figura 7: Melhoria em re-trabalho na Índia Gráfico de Controle - Antes e Depois.

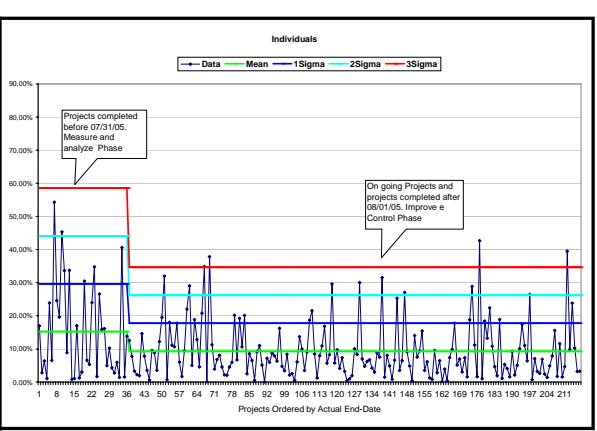

Figura 8: Melhoria em re-trabalho no Brasil- Gráfico de Controle - Antes e Depois.

\section{Considerações Finais}

Após a conclusão do projeto, pode-se observar que é viável utilizar o Six Sigma nos projetos de melhoria de processo promovidos na organização. Os resultados além de satisfatórios são incontestáveis, pois são baseados em dados.

Algumas lições aprendidas nesse projeto foram:

- O patrocinador precisa ter influência suficiente na organização para ajudar na implantação das melhorias.

- A equipe do projeto precisa ter conhecimento no assunto e alocação para se dedicar ao mesmo.

- A definição de métricas é a parte mais critica do projeto, pois sempre será preciso medir o antes e o depois no desenvolvimento de um projeto DMAIC.

- A definição do escopo é importante para evitar que o projeto tome proporções muito grandes.

- A medição precisa ser tomada de bases confiáveis.

- A análise de causas considerando o local e nas áreas de negócio foi importante para categorizar os problemas.

- Envolver todos os interessados na determinação das causas raiz é fundamental para garantir o patrocínio para a implementação das melhorias.

- Treinamento e compromisso das equipes são necessários em qualquer projeto, em um projeto geograficamente distribuído isso é mais importante ainda.

Os projetos Six Sigma ajudam na melhoria de processos de software e ajudam a atingir as metas propostas pelos objetivos estratégicos da organização, tornando as atividades de melhoria de processos alinhadas com os mesmos.

\section{Referências Bibliográficas}

Chrissis, Mary Beth; Konrad, Mike; Shrum, Sandy. (2003) "CMMI Guidelines for Process Integration and Product Improvement”. Edited by Addison-Wesley.

Goethert, Wolfhart; Fisher Matt. (2003) "Deriving Enterprise-Based Measures Using the Balance Scorecard and Goal-Driven Measurement Techniques”. Institute Carnegie Mellon University.

Niven, P.R. (2002) "Balance Scorecard step-by-step”. John Wile \& Sons.

Rocha, Ana R.; Maldonado, José; Weber, Kival. (2001) “Qualidade de Software - Teoria e Prática”. São Paulo, Prentice Hall. 